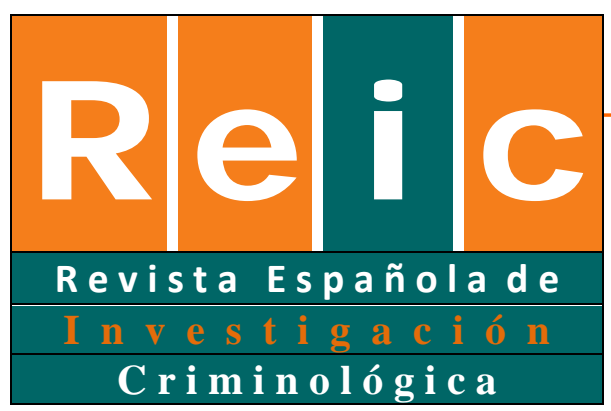

Crespi \& Mikulic

\title{
Estudio de la reinserción social de liberados condicionales desde un enfoque psicosocial
}

\section{Study of parolees' social reintegration from a psychosocial approach}

Recibido el 18 noviembre 2013/Publicado el 19 de marzo 2014

\author{
Melina Crespi \& Isabel M. Mikulic ${ }^{1}$ \\ Universidad de Buenos Aires
}

\begin{abstract}
RESUMEN
Este trabajo estudia la reinserción social de sujetos que han cumplido una condena y recobrado su libertad. Se comprende la reinserción social como un fenómeno que integra diversos niveles de análisis: micro, meso y macro. Participaron 160 liberados condicionales de la Ciudad de Buenos Aires, a los cuales se les administró los siguientes instrumentos: Entrevista para evaluar Perspectiva Futura, Cuestionario de Apoyo Social Comunitario, Escala de Prejuicio, Cuestionario de Reinserción Social, Inventario de Respuestas de Afrontamiento y Escala de Satisfacción Vital. El análisis de regresión múltiple mostró que las variables que mejor predicen la reinserción social de los liberados son: prejuicio social, respuestas de afrontamiento conductuales por aproximación, satisfacción vital, factibilidad de las metas de futuro, edad de inicio en el delito, actitud temporal futura y apoyo social. Estos resultados tienen implicaciones prácticas para la intervención psicosocial ligada a la problemática de reinserción y para la prevención de la reincidencia en el delito.
\end{abstract}

Palabras claves: modelo ecológico, reinserción social, liberados condicionales.

\footnotetext{
${ }^{1}$ La correspondencia debe enviarse a M. Crespi. Baigorria 3754, CP: 1417, Ciudad de Buenos Aires, Argentina.melinacrespi@yahoo.com.ar
} 


\begin{abstract}
This work studies the social reintegration of individuals who have served a sentence and regained their freedom. Social reintegration is understood as a phenomenon that integrates different levels of analysis: micro, meso and macro. 160 parolees of the City of Buenos Aires were administered the following instruments: Interview to assess Future Perspective, Community Social Support Questionnaire, Scale of Prejudice, Social Reintegration Questionnaire, Coping Responses Inventory and Life Satisfaction Scale. Multiple regression analysis showed that the variables that best predict the social reintegration of parolees are: social prejudice, behavioral coping responses by approach, life satisfaction, feasibility of future goals, age of onset in the crime, attitude future time and social support. These results have practical implications for psychosocial intervention linked to reintegration and the prevention of recidivism.
\end{abstract}

Key words: ecological model, reentry, parolees.

\title{
1. Introducción
}

En las últimas décadas el delito se ha convertido en la Argentina en uno de los principales problemas sociales. Tanto el inusitado interés que los medios prestan a este tema, como la sensación de inseguridad experimentada por la población, evidencian que el crecimiento del delito representa un hecho que genera una fuerte preocupación social.

De acuerdo a los datos proporcionados por la Dirección Nacional de Política Criminal del Ministerio de Justicia de la Nación, en los últimos veinte años la tasa de hechos delictivos en la totalidad del país se duplicó, pasando de 1.550 a 3.300 delitos cada 100.000 habitantes. La mayor parte de los hechos denunciados en Argentina corresponden a delitos contra la propiedad. El robo, incluyendo los casos de tentativa, explica el 52\% del total de delitos denunciados a nivel nacional. Otro aspecto importante de esta problemática está dado por el incremento en la cantidad de población privada de su libertad que se viene registrando en los últimos años. Según información del Sistema Nacional de Estadísticas sobre Ejecución de la Pena (SNEEP), en los últimos diez años la población carcelaria total en el país creció más del 80\%. A ello hay que agregar, de acuerdo al SNEEP, la existencia de una alta tasa de reincidencia, es decir, de un alto porcentaje de individuos sentenciados que delinquen nuevamente. 
Los aspectos señalados evidencian el déficit que encierran las políticas penitenciarias y postpenitenciarias cuyo marco legitimador se sustenta en una visión positiva de la pena, ligada a los supuestos de resocialización y reinserción social. Un interrogante que puede establecerse en relación a este aspecto está asociado al campo postpenitenciario y por lo tanto, al proceso por el que atraviesan quienes luego de haber cumplido una condena, obtienen su libertad. Numerosos estudios han mostrado que la transición que representa para un sujeto recobrar la libertad, constituye una situación altamente estresante en la que se retorna al contexto cotidiano quedando "libre", pero también "expuesto" (Visher y Travis, 2011). El impacto que genera esta situación se agudiza si consideramos la pobre intervención de los organismos encargados del proceso de reinserción de los liberados, así como la ausencia de políticas sociales que se ocupen de esta problemática.

A nivel teórico e investigativo, otro factor que agudiza el estado actual del fenómeno descripto, comprende la escasez de estudios e intervenciones estrictamente vinculadas al campo postpenitenciario. Si bien algunos trabajos abordan la cuestión en términos meramente teóricos, falta un tratamiento empíricamente riguroso que permita analizar la transición cárcel-libertad desde una perspectiva integral.

Considerando la necesidad de avanzar en el conocimiento de esta conflictiva, es que se propone en el presente estudio abordar el proceso de reinserción social de un grupo de sujetos que habiendo cumplido una condena ha obtenido la libertad condicional. Específicamente, desde un enfoque psicosocial, interesa poder analizar cómo diferentes variables, personales y contextuales, intervienen en dicho proceso generando mayores o menores posibilidades de inserción social. El conocimiento de cada una de ellas y de su injerencia en el proceso de reinserción, aportará datos empíricos relevantes para el desarrollo de estrategias de acción eficaces en el campo de asistencia penitenciaria y postpenitenciaria.

\section{Marco teórico}

Las problemáticas que estudiamos en ciencias sociales suelen ser extremadamente complejas dado que se encuentran matizadas por un gran número de variables y factores interdependientes. Esto impide que puedan ser abordadas en su totalidad y 
fundamentalmente establecer con exactitud cuál es la causa y cuál el efecto, en tanto que, causas y efectos se mezclan mediante un complejo proceso de retroalimentación.

La problemática delictiva como fenómeno psicosocial, y en relación a ella el fenómeno de la reinserción social, se enmarca dentro de esta complejidad y exige en este sentido estudiar el intrincado interjuego de factores que están en la base del mismo (Mikulic \& Crespi, 2007). Ello supone la necesidad de poner en cuestión los modos instituidos con los que comúnmente se piensa y se interviene en relación al proceso de reinserción social de quienes han recobrado su libertad. En efecto, dicho proceso ha sido tradicionalmente considerado a través de abordajes que han tendido a centrarse en forma exclusiva o bien, en las características individuales de quienes delinquen, o bien, en un conjunto de factores socio-económicos e institucionales capaces de favorecer u obstaculizar dinámicas de integración social.

Si bien, se reconoce la importancia de los distintos factores mencionados, la adopción de una perspectiva restringida a cierto tipo de variables, ha sido un aspecto fuertemente cuestionado por diversos autores (Kelly, 1992; Prilletenky, 2000; Castellá, 2008). Ello ha dado paso al desarrollo de nuevos modelos conceptuales que, desplazando y ampliando la mirada desde el individuo al contexto social, enfatizan la interdependencia entre distintos niveles de análisis. De estos modelos cabe destacar el Enfoque Ecológico, cuyos supuestos epistemológicos y metodológicos, constituyen el marco de estudio de la presente investigación. La adopción de este enfoque se fundamenta en la importancia de poder abordar la conflictiva de reinserción social mediante la consideración de múltiples sistemas, ecológicamente conectados.

Uno de los autores más representativos de esta perspectiva es Bronfrenbrenner (1987), quien propone una “Ecología del Desarrollo Humano“. Para el autor, el ambiente ecológico en el que los individuos se desempeñan representa un sistema dinámico, en el que se establecen pautas de interacción recíprocas entre el sujeto activo y su entorno físico y social. Cuatro niveles o estructuras concéntricas íntimamente relacionadas conforman dicho ambiente ecológico: a) el microsistema, considerado como el nivel más cercano al sujeto, donde se producen las interacciones cara a cara; b) el mesosistema, centrado en las interrelaciones que existen entre dos o más entornos en los que la persona participa activamente (familia, trabajo y vida social); c) el exosistema, que también condensa las relaciones entre dos o más entornos, pero éstos no incluyen a 
la persona como participante activo; y d) el siguiente nivel, el macrosistema, integrado por los valores culturales, las creencias, las representaciones que rigen en una sociedad en un momento particular. Desde el modelo propuesto por Bronfenbrenner es posible así una compresión ecológica-sistémica de la realidad que contempla la riqueza multidimensional de los fenómenos psicosociales (Castellá, 2008).

Otro autor de gran importancia es Belsky (1993), quien desde un marco evolutivo propone la integración de una serie de sistemas cada vez más amplios. Al igual que Bronfenbrenner (1987), para Belsky el medio ecológico que caracteriza el intercambio entre las personas y sus ambientes, se compone de estructuras físicas, sociales y psicológicas. Este medio ecológico es concebido como un conjunto de estructuras concéntricas de mutua influencia que el autor divide en tres contextos principales: a) el macrosistema, en tanto nivel más general que incluye las formas de organización social, las creencias y los estilos de vida propios de una cultura o subcultura específica; b) el exosistema, que incluye el nivel de relaciones más próximas de las personas. Tales relaciones están mediatizadas por un conjunto de instituciones que operan entre la cultura y el nivel individual (escuela, organismos de control social, iglesia, centros de salud, entre otros); c) el microsistema, compuesto por las relaciones más próximas entre las personas, donde Belsky (1993) agrega además el nivel del ontosistema, comprendiendo los factores individuales.

Estudiar desde esta perspectiva la reinserción social de quienes han sido privados de su libertad, requiere la integración de los distintos niveles mencionados:

(i) El nivel micro o individual, se asocia en este estudio con las variables respuestas de afrontamiento implementadas ante eventos estresantes, perspectiva temporal futura y nivel de satisfacción vital percibido. También se incluyen factores de trayectoria delictiva y penitenciaria, como edad de inicio en el delito y tiempo de condena cumplido.

Diversas investigaciones han demostrado el papel relevante que cada una de estas variables tiene en la reinserción social del liberado. Considerando el constructo afrontamiento, se hipotetiza que la puesta en marcha de determinadas estrategias de afrontamiento son útiles para una adecuada resolución de conflictos en el período de egreso carcelario. Se ha advertido que el uso de estrategias de afrontamiento activas, 
como la búsqueda de apoyo y el análisis lógico, representan factores de protección que discriminan entre quienes vuelven a reincidir luego de una condena y quienes no reinciden (Carr \& Vandiver, 2001; Crespi \& Mikulic, 2004; Blasco, 2012). Otra variable sumamente importante que incluimos en este nivel de análisis, refiere a la perspectiva temporal futura (PTF). Distintos estudios coinciden en señalar que las personas que han cometido delitos tienen una perspectiva de tiempo más corta y metas más orientadas al presente, respecto de los no delincuentes (Trommsdorff, 1994; Black \& Grecson, 2011; Clinkinbeard, 2013). Trommsdorff \& Lamm (2006) al estudiar la PTF en delincuentes institucionalizados y no delincuentes, observaron que las principales diferencias se advierten en la orientación temporal y en el interés asignado al futuro. Los primeros evaluaron su pasado y su futuro como más negativo y mostraron menor interés respecto al futuro. En esta línea, otros estudios realizados con personas que luego de haber cumplido una condena volvían a reincidir y no reincidentes, permitió observar que las variables elaboración de un proyecto de futuro y sentido de vida, representaban factores de protección que diferenciaban entre los grupos, a favor de los no reincidentes (Crespi \& Mikulic, 2004; Black \& Grecson, 2011). Estrechamente asociada a la PTF, la variable satisfacción vital ha mostrado también diferencias significativas entre grupos de reincidentes y no reincidentes. De acuerdo a ello, una pobre valoración de la propia vida ha tendido a asociarse con mayor reincidencia en el delito (Heisel \& Flett 2004; Kim \& Kim 2008; Martin \& Stermac, 2010; Fulya \& Yasin, 2012). Por el contrario, distintos autores reconocen que la satisfacción vital constituye un factor de resiliencia o de desistimiento vinculado a la reducción exitosa de la actividad delictiva (Lösel, 2010; Laws \& Ward, 2011; Ward, Rose \& Willis, 2012).

Respecto a las variables criminológicas incluidas en el modelo ecológico propuesto, son numerosos los estudios que han demostrado el efecto negativo que las variables, edad de inicio en el delito y tiempo de condena tienen, en la reinserción social del liberado. Las mismas han tendido a asociarse positivamente con el mantenimiento posterior de la conducta delictiva (Vázquez, 2003; Bottoms \& Shapland, 2011; Bringas, Rodríguez, Moral, Pérez \& Ovejero, 2012; Piquero, Hawkins, Kazemian, Petechuk \& Redondo, 2013). 
(ii) El nivel meso o relacional propuesto, incluye el análisis de las características estructurales y funcionales de las redes sociales de apoyo de los liberados. Este es un aspecto central en la medida en que aporta a la comprensión de los procesos de integración social, o por el contrario de vulnerabilidad psicosocial. Como señalan Bain \& Parkinson (2010), el éxito de la reinserción social depende y es proporcional al grado de vinculación social del individuo. Incluso, existe evidencia empírica que demuestra que el apoyo social es un factor central ligado al desistimiento delictivo (Cid \& Martí, 2011, 2012). Como afirman Cid \& Martí (2012), los vínculos que surgen con la familia nuclear o con alguna pareja en el período de egreso, suelen adquirir un rol significante en la narrativa del desistimiento. Lösel (2010) señala que algunos de los factores centrales que intervienen en el desistimiento delictivo comprenden: la presencia de una relación estable con al menos una persona de referencia, un adecuado nivel de apoyo social y la existencia de modelos sociales que promuevan un tipo de afrontamiento constructivo. Otros autores ponen el acento en la ocurrencia de determinados sucesos vitales ligados a cambios en las redes de apoyo, tales como: presencia de una pareja, inserción laboral, alejamiento de pares problemáticos, tener hijos, o la participación en organizaciones sociales (Laub \& Sampson, 2003; Uggen, Manza \& Behrens, 2004). Por su parte, Casey \& Jarman (2011) se centran en el rol central que la familia tiene en el proceso de reinserción social, señalando la necesidad de favorecer los vínculos con el entorno familiar durante el período de encarcelamiento. En esta línea, Visher \& Travis (2011) afirman que las posibilidades de una reinserción social efectiva dependen no solamente de las actitudes y expectativas del liberado, sino también del rol de la familia y la red comunitaria, al aportar apoyo u obstaculizar dicho proceso.

(iii) Por último, el macrosistema se asocia en este estudio a las variables percepción de ser objeto de prejuicio por parte de la sociedad y de los organismos de control social. La inclusión de estos indicadores se fundamenta en una serie de criterios adoptados para tal fin. En primer término, su elección deriva del relevamiento bibliográfico efectuado. Diversas investigaciones que han aplicado el enfoque ecológico para la comprensión de problemáticas complejas, como la enfermedad mental (Rodgers, 2009), la psicooncología (Torrico, Santín, Montserrat, Menéndez \& López, 2002), la discapacidad (Sobsey, 1994; Céspedes, 2005) y en particular, la problemática 
delictiva (Valdenegro, 2005), han tomado la variable prejuicio como uno de los factores centrales del macrosistema. Por otro lado, se han tomando en cuenta las definiciones que aportan diversos exponentes del enfoque ecológico sobre el nivel macro. Así, Belsky (1993) y Prilletenky (2000) lo definen como un subsistema del ambiente ecológico en el que los individuos se desarrollan. Dicho subsistema abarca los patrones culturales, las significaciones sociales, creencias y actitudes que rigen en una sociedad en un momento histórico particular. Por su parte, Bronfenbrenner (1987) agrega que el macrosistema no sólo comprende las macroinstituciones (gobierno, sistema económico, políticas públicas, mercado de trabajo, etc.), sino también los aspectos actitudinales, las creencias y los estereotipos que predominan en una sociedad en un momento dado. Desde este nivel, entendemos que son muchos los factores que pueden tener injerencia en el proceso de reinserción de las personas que han recobrado su libertad. Sin embargo, como reconocen distintos autores, uno de los más significativos esta dado por las barreras actitudinales que el liberado encuentra por su condición de tal (Dodge \& Pogrebin, 2001; Ross \& Richards, 2003; Valdenegro, 2005; Villagra, 2008). En el macrosistema, las actitudes y las creencias hacia la delincuencia y la reinserción social de los liberados, son importantes para promover u obstaculizar dinámicas de integración comunitaria. En tal sentido, el macrosistema se comprende vinculado a la variable percepción de ser objeto de prejuicio, como reflejo de los estereotipos culturales acerca de la delincuencia, y las oportunidades que de allí se derivan en términos de posibilidades de inserción social. Las actitudes que el sujeto percibe en sus entornos cotidianos están en buena parte, en función de la imagen más general que la sociedad tenga de la delincuencia -sustentada en gran medida por el tratamiento que le dan diversas instancias, como es el caso de los medios de comunicación-.

Por otro lado, las actitudes y respuestas sociales que el sujeto percibe, repercuten en los otros subsistemas ecológicos en los que participa. Como se sostiene desde el enfoque ecológico, el macrosistema ejerce efectos sobre los distintos subsistemas. De esta manera, la existencia de determinadas creencias y actitudes sociales acerca de la delincuencia (nivel macro), incide en los recursos de apoyo disponibles en el contexto social (nivel meso) y en el entorno más íntimo (nivel micro) (Angenent \& Man, 1996; Frías, López \& Díaz, 2003; Valdenegro, 2005; Crespi, 2012). 
En base al modelo ecológico descripto se proponen como objetivos de este estudio:

$\left(1^{\circ}\right)$ Evaluar la asociación existente entre las variables consideradas en los distintos niveles de análisis destacados (micro, meso y macrosistema) y la reinserción social de aquellas personas que han recobrado su libertad.

$\left(2^{\circ}\right)$ Analizar la capacidad predictiva que las variables asociadas al modelo ecológico propuesto tienen sobre la reinserción social del liberado.

\section{Método}

\subsection{Participantes}

Se seleccionó una muestra intencional conformada por 160 sujetos que han cumplido condena y se encuentran en libertad condicional, entre las edades de 18 y 45 años. El tamaño muestral se estimó considerando las exigencias de los métodos estadísticos propuestos para responder a los objetivos del estudio, siguiendo las recomendaciones de distintos autores (Nunnally, 1991; Green, 1991; Tabachnick \& Fidell, 2001).

La investigación se desarrolló en un Juzgado de Ejecución Penal y en el Patronato de Liberados de la Ciudad de Buenos Aires, Argentina.

Para la selección de los participantes se han controlado las variables género, edad, tipo de delito y tiempo de egreso, en tanto entendemos que el proceso de reinserción puede variar de manera significativa en función de tales factores. Así, la totalidad de los participantes entrevistados fueron hombres, el tipo de delito corresponde a delitos contra la propiedad, principalmente robo calificado, y el tiempo de egreso o de obtención de la libertad condicional no superaba los seis meses. Se seleccionó este plazo, en la medida en que diversos estudios coinciden en señalar que los primeros seis meses después de la liberación son las más proclives para la reincidencia, en virtud de las diversas situaciones estresantes que el liberado atraviesa durante el proceso de su reinserción al medio social (García, 1991; Colmenarez, 2005; Ibañez \& Cid, 2013).

Dentro del rango estudiado (18 a 45 años) la media de edad es de 30 años (SD= 6). El $47 \%$ de los sujetos son solteros, mientras que el restante se polariza entre un $25 \%$ 
en concubinato, un 21,5\% separado y un 6,5\% casado. Con respecto al nivel de escolaridad, el mayor porcentaje se concentra en la categoría de estudio primario completo (53\%), seguido de secundario incompleto (33,6\%).

El 63\% de los evaluados reconoce haber cometido por lo menos un delito anterior al hecho actual por el cual ha sido condenado. Estos antecedentes se ubican en un rango de edad muy temprano, entre los 11 y 23 años, concentrándose la mayor cantidad de los mismos en el rango de los 14 y 16 años. Los delitos cometidos con anterioridad corresponden a delitos contra la propiedad, principalmente robo simple (42\%), seguido del robo calificado (40,5\%). Respecto al tipo de delito por el cual los participantes han cumplido condena, tal como se mencionó, se trata de delitos contra la propiedad. Dentro de esta categoría el robo calificado (68\%) y el robo simple (24\%) son los que asumen la mayoría de los casos $^{2}$.

\subsection{Instrumentos y variables}

A fin de evaluar cada una de las variables del modelo ecológico propuesto se han construido y/o adaptado los siguientes instrumentos:

\section{Variables Independientes}

\section{(i) Cuestionario socio-demográfico y criminológico}

Ha sido diseñado con el fin de recabar información sobre características sociodemográficas de los entrevistados (edad, nivel educativo, estado civil, etc.) y sobre distintas variables ligadas a la historia criminológica (edad de inicio en el delito, tipo de delito, tiempo de condena, etc.).

(ii) Entrevista para evaluar Perspectiva Temporal Futura

\footnotetext{
${ }^{2}$ Cabe señalar que la distinción entre robo simple y calificado, se establece en función de la gravedad del hecho y de la consecuente pena que corresponde en cada caso. Mientras que el robo simple supone el apoderamiento ilegítimo de una cosa mueble ajena, con fuerza en las cosas o con violencia física en las personas; el robo calificado implica otros agravantes, como el uso de armas o el ser cometido en despoblado y en banda. Por tal razón, la pena que corresponde a éstos últimos es mayor que en los casos de robo simple (Arts. 164 y 166, Código Penal Argentino).
} 
Este instrumento se construyó tomando en consideración el modelo teórico propuesto por Nurmi (1994). De acuerdo al mismo, la Perspectiva Temporal Futura representa un fenómeno complejo y multidimensional, en el que intervienen tres procesos: la motivación, el planeamiento y la evaluación. El cuestionario evalúa tales procesos a partir de la descripción de las metas que los sujetos desean lograr y de su extensión o ubicación temporal (motivación), las modalidades imaginadas y concretas a través de las cuales se planea la realización de las mismas (planificación), la probabilidad de logro percibida y el locus de control externo o interno (evaluación prospectiva).

Para la evaluación de estas dimensiones de la Perspectiva Temporal Futura se realizan preguntas con respuesta libre en el caso de la identificación de metas (¿Qué metas u objetivos que le gustaría alcanzar?), como así también en la identificación de acciones planificadas y ejecutadas (¿Qué haría para alcanzar esas metas? ¿Qué acciones está realizando para alcanzarlas?).

Para la dimensión de orientación o extensión temporal se solicita a los participantes que definan en cuánto tiempo esperan alcanzar cada una de las metas identificadas y que asignen esa cantidad de tiempo a una de las siguientes categorías: corto plazo (futuro cercano), largo plazo (futuro distante) y de ahora en adelante (presente abierto).

Las variables probabilidad de logro y locus de control, las cuales se agrupan en torno a la evaluación prospectiva, se evalúan en la entrevista a partir de preguntas que admiten cuatro opciones de respuesta y la justificación de elección por parte del entrevistado.

La entrevista integra asimismo la dimensión de actitud temporal, referida a la valoración del pasado, presente y futuro. Para la evaluación de las actitudes temporales se construyó una escala tipo likert que incluye una serie de afirmaciones frente a las cuales los participantes seleccionan una de cinco opciones o gradaciones de respuestas que van de Nunca a Siempre. Se evaluó la confiabilidad de la misma, a partir el método alfa de Cronbach. Los valores alfa obtenidos han sido adecuados para cada una de las dimensiones de la actitud temporal considerados: .70 para actitud hacia el pasado, .75 para actitud hacia el presente, y .78 para actitud hacia el futuro. 
(iii) Inventario de Respuestas de Afrontamiento (Moos, 1993, adaptación Mikulic, 1998)

Esta prueba consta de 48 ítems distribuidos en ocho escalas específicas, cuatro de las cuales evalúan estrategias de afrontamiento por aproximación (dos cognitivas y dos conductuales) y las otras cuatro estrategias de afrontamiento por evitación (divididas también en cognitivas y conductuales). Los valores alfa obtenidos para cada una de las escalas mencionadas son: .72 para la escala de estrategias de aproximación cognitivas, .70 para las estrategias de aproximación conductuales, .73 en el caso de las estrategias de evitación cognitivas y .70 para las estrategias de evitación conductuales.

(iv) Escala de Satisfacción con la Vida (Pavot \& Diener, 1993, adaptación Mikulic, Cassullo \& Crespi, 2009)

Esta Escala permite evaluar la valoración global que la persona hace sobre su vida, comparando sus logros con sus expectativas. Consiste en un conjunto de afirmaciones frente a las cuales los sujetos deben indicar su nivel de acuerdo o desacuerdo en una escala de 7 puntos. La consistencia interna obtenida en este estudio a través del coeficiente alfa de Cronbach fue de .88, valor similar al obtenido en otras investigaciones (Pavot \& Diener, 1993; Martínez, 2004).

\section{(v) Cuestionario de Apoyo Social Comunitario}

Ha sido construido con el fin de evaluar el apoyo social en sus aspectos estructurales (tamaño de la red, frecuencia de contactos, reciprocidad, etc.) y funcionales (apoyo psicológico, informacional e instrumental), considerando tres niveles de apoyo: relaciones íntimas, sociales y comunitarias. También se indaga sobre el grado de satisfacción con el apoyo recibido.

El formato de los reactivos incluye:

a) preguntas abiertas para la evaluación de los aspectos estructurales del apoyo. Por ejemplo, para evaluar el tamaño de la red en el nivel de relaciones íntimas, se le solicita al sujeto que piense en aquellas personas que forman parte de su entorno más 
cercano, aquellas con las que tiene un vínculo de confianza y que responda a las siguientes preguntas:

- ¿Considera que ante una situación problemática esas personas podrían apoyarlo?

- En caso afirmativo, ¿Cuáles son las personas de su entorno más cercano que podrían ayudarlo? Menciónelas.

El mismo procedimiento se adopta para evaluar el tamaño de la red considerando el nivel social (personas que si bien no forman parte del entorno más cercano, tienen alguna relación con el sujeto, directa o indirectamente: amigos, conocidos, compañeros de trabajo, parientes lejanos, etc.) y el nivel comunitario (personas e instituciones de su vecindario o comunidad, por ejemplo, la iglesia, ONGs, centro de salud, patronato, etc.).

b) 54 ítems (afirmaciones) con formato de respuesta tipo likert para evaluar los aspectos funcionales. Por cada nivel de análisis (relaciones íntimas, sociales y comunitarias) se solicita al evaluado que indique con qué frecuencia ante una situación problemática las personas y/o instituciones que mencionó previamente, harían por ejemplo, las siguientes acciones:

- Escucharían mis problemas (Nunca-Poco-A veces-Bastante-Siempre)

- Me prestarían dinero si lo necesitara (Nunca-Poco-A veces-Bastante-Siempre)

-Podrían darme información útil para resolver algún problema (Nunca-Poco-A veces-Bastante-Siempre).

Considerando la confiabilidad del instrumento, para las escalas de Apoyo Psicológico, Instrumental e Informacional en el nivel de Relaciones Intimas los valores alfa obtenidos han sido: .89, .90 y .89 respectivamente. En el nivel de relaciones sociales los valores fueron: .90 (apoyo psicológico), .89 (apoyo instrumental) y .90 (apoyo informacional). $\mathrm{Y}$ en el nivel de relaciones comunitarias los valores obtenidos han sido: .91 (apoyo psicológico), .87 (apoyo informacional) y .87 (apoyo instrumental). 
(vi) Escala de Prejuicio Social

Este instrumento ha sido especialmente construido en este estudio con el propósito de evaluar el grado en que los sujetos se autoperciben como objeto de prejuicio, considerando dos fuentes principales: la sociedad y el control social (policía). La prueba consta de 18 ítems consistentes en afirmaciones ante las cuales los sujetos señalan su grado de acuerdo en una escala que va de Nunca a Casi siempre. Los coeficientes alfa obtenidos son adecuados: 92 (escala percepción de ser objeto de prejuicio por parte de la sociedad) y .89 (escala percepción de ser objeto de prejuicio por parte de la policía).

\section{Variable dependiente}

\section{(i) Cuestionario de Reinserción Social}

Ha sido construido a fin de evaluar distintas dimensiones ligadas al proceso de reinserción social. Se entiende la reinserción como un constructo multidimensional que comprende tanto el grado de acceso y participación del sujeto en diversos ámbitos de la vida social (trabajo, educación, salud, espacios comunitarios); como la disponibilidad de recursos materiales que aseguren adecuadas condiciones de vida (dimensión material) (Laparra, 1999). Con el fin de operativizar el concepto, se han identificado sus dimensiones principales y sus respectivos indicadores, tal como se presenta a continuación:

a) Condiciones de Vivienda: disponibilidad de vivienda, forma de tenencia, tipo de vivienda, material, cantidad de personas por hogar, cantidad de habitaciones, dificultades registradas en la vivienda, disponibilidad de agua potable, disponibilidad de luz artificial, capacidad de mantenimiento.

b) Situación Laboral: inserción actual en actividades laborales, tipo de trabajo, horas semanales, tipo de relación laboral, estabilidad laboral, satisfacción con el empleo. Déficit de empleo (desocupación, precariedad laboral).

c) Situación Económica: nivel de ingresos, procedencia de los ingresos, hábitos de consumo (privaciones como consecuencia de la situación económica), nivel de ingreso considerando el total de personas con las que se convive, apoyo o ayuda social, 
posibilidad de cubrir gastos mínimos necesarios de subsistencia.

d) Situación Educacional: nivel de instrucción alcanzado, inserción en actividades educativas o de formación ocupacional, características, factores asociados a la inserción educacional o a las dificultades percibidas en relación a este aspecto.

e) Condiciones sanitarias: problemas de salud, acceso y uso de servicios sanitarios, características, disponibilidad de recursos económicos para costear tratamientos o medicación, pertenencia a obra social, satisfacción con los servicios recibidos.

f) Participación Social: participación en organizaciones sociales, recreativas, políticas, barriales, religiosas, etc. Forma y grado de participación, interés en participar.

El cuestionario aporta un puntaje por cada una de las dimensiones consideradas. Asimismo, a partir de la aplicación de un análisis factorial exploratorio, se puso en evidencia que las dimensiones mencionadas se agrupan en dos factores denominados: inserción social y condiciones materiales. De la sumatoria de las puntuaciones de cada uno de los factores identificados se obtiene un puntaje total (índice de reinserción social). Respecto a la confiabilidad de la prueba, los valores alfa obtenidos han sido adecuados para cada uno de los factores: .79 para el factor inserción social y .70 para el factor condiciones materiales.

Sintetizando, las variables que se integran en este estudio se presentan en el siguiente cuadro: 
Cuadro 1. Variables de la investigación

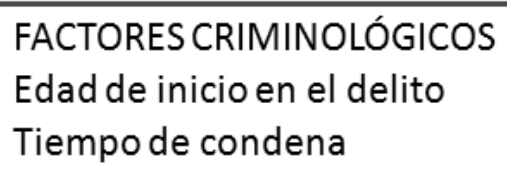

\section{PTF}

Cantidad de metas

Probabilidad de logro metas

Locus de control

Actitud temporal pasada

Actitud temporal presente

Actitud temporal futura

\section{AFRONTAMIENTO}

Afrontamiento por aproximación

cognitivo

Afrontamiento por aproximación

conductual

Afrontamiento por evitación cognitivo

Afrontamiento por evitación conductual

\section{SATISFACCIÓN VITAL}

Nivel de satisfacción vital
APOYO SOCIAL COMUNITARIO

Tamaño de la red de apoyo

Reciprocidad

Apoyo psicológico relaciones íntimas

Apoyo instrumental relaciones íntimas

Apoyo informacional relaciones íntimas

Total apoyo relaciones intimas

Apoyo psicológico relaciones sociales

Apoyo instrumental relaciones sociales

Apoyo informacional relaciones sociales

Total apoyo relaciones sociales

Apoyo psicológico nivel comunitario Apoyo instrumental nivel comunitario

Apoyo informacional nivel comunitario

Total apoyo comunitario

Satisfacción con el apoyo recibido

PERCEPCIÓN DE PREJUICIO

Percepción de ser objeto de prejuicio

por parte de la sociedad

Percepción de ser objeto de prejuicio

por parte de la policía

\section{INDICE DE REINSERCIÓN SOCIAL}

(inserción social y condiciones materiales)

\subsection{Procedimiento}

Los instrumentos mencionados se administraron a personas en situación de libertad condicional. La investigación se efectuó en el marco de un dispositivo de asistencia e intervención llamado Clínica de la Vulnerabilidad que funciona en un Juzgado de Ejecución Penal de la Ciudad Autónoma de Buenos Aires, y que tiene por objeto la implementación de medidas y estrategias de abordaje interdisciplinario que posibiliten una mejor transición cárcel-libertad. También se recurrió al Patronato de Liberados de Ciudad Autónoma de Buenos Aires y se realizaron entrevistas a población tutelada que 
asiste mensualmente a tal organismo. En primer término se explicó a los participantes los propósitos de la investigación, se solicitó su consentimiento para colaborar y se señaló el carácter confidencial y anónimo de los resultados obtenidos. Los instrumentos de evaluación reseñados anteriormente se aplicaron en forma individual, en dos sesiones, bajo las mismas condiciones para la muestra de participantes. El tiempo total por sesión insumió entre 45 y 60 minutos.

\section{Resultados}

\subsection{Análisis de Correlación}

Se realizó en primera instancia un análisis de correlación entre las variables incluidas en el modelo ecológico propuesto con el propósito de seleccionar aquellas que presentan mayor asociación con la reinserción social, y en función de ello, realizar posteriormente un análisis conjunto de las mismas mediante el método de regresión múltiple.

Tal como se aprecia en la Tabla 1, el análisis de correlaciones bivariadas muestra la existencia de asociaciones positivas significativas entre la reinserción social y diversas variables del nivel micro considerado en este estudio.

Tabla 1. Correlaciones entre Reinserción Social y variables del nivel micro del Modelo Ecológico

\begin{tabular}{|l|l|}
\hline & Reinserción Social \\
\hline Edad de inicio en delito & $.46^{* *}$ \\
\hline Tiempo de condena & .02 \\
\hline Satisfacción vital & $.42^{* *}$ \\
\hline Cantidad de metas & $.20^{*}$ \\
\hline Probabilidad de logro & $.33^{* *}$ \\
\hline Locus de control & .15 \\
\hline Actitud temporal pasada & $.30^{* *}$ \\
\hline Actitud temporal presente & $.34^{* *}$ \\
\hline Actitud temporal futura & $.32^{* *}$ \\
\hline Afrontamiento por aproximación cognitivo & $.37^{* *}$ \\
\hline Afrontamiento por aproximación conductual & $.36^{* *}$ \\
\hline Afrontamiento por evitación cognitivo & -.18 \\
\hline Afrontamiento por evitación conductual & -.17 \\
\hline
\end{tabular}


Nota: $* * p<.01, * p<.05$

Considerando las variables incluidas en el nivel relacional del modelo ecológico propuesto, se observan asociaciones positivas significativas entre el nivel de reinserción social y diversas características del apoyo social, tal como se detalla en la Tabla 2.

Tabla 2. Correlaciones entre Reinserción Social y variables del nivel relacional del Modelo Ecológico

\begin{tabular}{|l|l|}
\hline & Reinserción Social \\
\hline Tamaño de la red & $.22^{*}$ \\
\hline Reciprocidad & .08 \\
\hline Apoyo psicológico relaciones íntimas & $.24^{* *}$ \\
\hline Apoyo instrumental relaciones íntimas & .19 \\
\hline Apoyo informacional relaciones íntimas & $.30^{* *}$ \\
\hline Total apoyo relaciones íntimas & $.28^{* *}$ \\
\hline Apoyo psicológico relaciones sociales & $.22^{*}$ \\
\hline Apoyo instrumental relaciones sociales & .17 \\
\hline Apoyo informacional relaciones sociales & .09 \\
\hline Total apoyo relaciones sociales & .18 \\
\hline Apoyo psicológico nivel comunitario & .03 \\
\hline Apoyo instrumental nivel comunitario & .02 \\
\hline Apoyo informacional nivel comunitario & .07 \\
\hline Total apoyo nivel comunitario & .02 \\
\hline Satisfacción con el apoyo & $.20^{*}$ \\
\hline
\end{tabular}

Nota: $* * p<.01, * p<.05$

Por último, desde el nivel macro, se evidencian correlaciones negativas significativas entre la reinserción social y las variables percepción de ser objeto de prejuicio por parte de la sociedad y de la policía (Tabla 3).

Tabla 3. Correlaciones entre Reinserción Social y Percepción de ser objeto de prejuicio

\begin{tabular}{|l|l|}
\hline & Reinserción Social \\
\hline Percepción de prejuicio por parte de la sociedad & $-.55^{* *}$ \\
\hline Percepción de prejuicio por parte de la policía & $-.61^{* *}$ \\
\hline
\end{tabular}

Nota: ${ }^{* *} p<.01$, * $p<.05$ 


\subsection{Análisis de regresión}

Para determinar cuál o cuáles de las variables psicosociales estudiadas predicen de mejor manera la reinserción social, se llevó a cabo un análisis de regresión lineal múltiple mediante el procedimiento stepwise (pasos sucesivos). Esta técnica estadística es sumamente útil, pues permite analizar datos que provienen de problemas complejos, en los que intervienen multiplicidad de variables (Camarillo \& García, 2003).

En la ecuación matemática de regresión múltiple especificada, la variable reinserción social tomó el papel de variable dependiente o criterio, mientras que las demás variables del modelo ecológico reseñado actuaron como variables predictoras. En la Tabla 4, se aprecian los coeficientes de correlación múltiple $\left(R^{2}\right)$ de cada modelo entre las variables predictoras y la variable criterio. El primer modelo obtuvo un $R^{2}=$ .37, luego, en los pasos sucesivos se han introducido las demás variables predictoras, hasta llegar al modelo 7 en que todas las variables se han correlacionado con la variable criterio con un $R^{2}=.56$. Las variables que mejor predicen la reinserción social de los liberados son: en un sentido negativo, la percepción de ser objeto de prejuicio social por parte de la sociedad; y en un sentido positivo, las respuestas de afrontamiento por aproximación, en particular las conductuales, la satisfacción vital, la probabilidad de logro de las metas de futuro, la edad de inicio en el delito, la actitud temporal futura y el apoyo social proveniente de las relaciones íntimas (apoyo psicológico, instrumental e informacional). Estas variables juntas explican un 56\% de la varianza total de las puntuaciones de los sujetos $\left(R^{2}=.56 ; \mathrm{F}=30.04 ; \mathrm{p}=.000\right)$. 
Tabla 4. Análisis de regresión lineal múltiple por pasos sucesivos: Variable criterio Reinserción Social

\begin{tabular}{|c|c|c|c|c|}
\hline Modelo & $\boldsymbol{\beta}$ & $\mathbf{t}$ & $\mathbf{p}$ & $\mathbf{R} 2$ \\
\hline Paso 1 & & & & .37 \\
\hline Prejuicio social & $-.61 * * *$ & -9.84 & .00 & \\
\hline Paso 2 & & & & .43 \\
\hline Prejuicio social & $-.56 * * *$ & -9.16 & .00 & \\
\hline Aproximación conductual & & 3.87 & .00 & \\
\hline Paso 3 & & & & .47 \\
\hline Prejuicio social & $-.48 * * *$ & -7.60 & .00 & \\
\hline Aproximación conductual & $.23 * * *$ & 3.88 & .00 & \\
\hline Satisfacción vital & $.22 * * *$ & 3.55 & .00 & \\
\hline Paso 4 & & & & .51 \\
\hline Prejuicio social & $-.44 * * *$ & -7.15 & .00 & \\
\hline Aproximación conductual & $.20 * *$ & 3.49 & .00 & \\
\hline Satisfacción vital & $.23 * * *$ & 3.93 & .00 & \\
\hline Probabilidad de logro metas & $.21 * * *$ & 3.73 & .00 & \\
\hline Paso 5 & & & & .54 \\
\hline Prejuicio social & $-.36 * * *$ & -5.72 & .00 & \\
\hline Aproximación conductual & $.16 * * *$ & 3.01 & .00 & \\
\hline Satisfacción vital & $.22 * * *$ & 3.89 & .00 & \\
\hline Probabilidad de logro metas & $.21 * * *$ & 3.90 & .00 & \\
\hline Edad de inicio en delito & $.20 * * *$ & 3.49 & .00 & \\
\hline Paso 6 & & & & .55 \\
\hline Prejuicio social & $-.36 * * *$ & -5.84 & .00 & \\
\hline Aproximación conductual & $.19 * * *$ & 3.39 & .00 & \\
\hline Satisfacción vital & $.24 * * *$ & 4.22 & .00 & \\
\hline Probabilidad de logro metas & $.21 * * *$ & 3.99 & .00 & \\
\hline Edad de inicio en delito & $.21 * * *$ & 3.67 & .00 & \\
\hline Actitud temporal futura & $.11^{*}$ & -2.03 & .03 & \\
\hline Paso 7 & & & & .56 \\
\hline Prejuicio social & $-.33 * * *$ & -5.31 & .00 & \\
\hline Aproximación conductual & $.19 * * *$ & 3.42 & .00 & \\
\hline Satisfacción vital & $.22 * * *$ & 3.86 & .00 & \\
\hline Probabilidad de logro metas & $.23 * * *$ & 4.24 & .00 & \\
\hline Edad de inicio en delito & $.19 * * *$ & 3.23 & .00 & \\
\hline Actitud temporal futura & $.14 * *$ & -2.40 & .01 & \\
\hline Total apoyo relaciones íntimas & $.13^{*}$ & 2.21 & .02 & \\
\hline
\end{tabular}

Nota: $* * * p<.001, * * p<.01, * p<.05$ 
El índice de tolerancia de las variables incluidas en la ecuación de regresión presenta valores comprendidos entre .70 y .95, y entre 1.05 y 1.48 en el caso del índice FIV. Asimismo no hay correlaciones mayores a .90 entre ninguna de las variables independientes y no hay presencia simultánea de betas mayores que 1 y menores que -1 . Por lo expuesto, la probabilidad de error derivada de la posible multicolinealidad entre las variables no supone un problema importante en la interpretación de los resultados (Bisquerra, 1989).

\section{Discusión}

\subsection{Conclusiones}

Los factores que intervienen en el proceso de reinserción al contexto cotidiano y social de quienes han recobrado su libertad luego de una condena, son variados y complejos. Además, se sitúan a diversos niveles y pueden ejercer su influencia de una forma directa y/o indirecta sobre el liberado, manteniendo importantes interconexiones entre sí. Esto hace que el proceso de reinserción social deba ser evaluado y comprendido como un fenómeno complejo, en el que se intrincan múltiples variables. La Perspectiva Ecológica es particularmente útil en tal sentido, en tanto representa un modelo de evaluación que permite pasar de un enfoque centrado en el sujeto, hacia uno basado en la relación sujeto-contexto como unidad de análisis indisociable. En el presente estudio se ha pretendido abordar la problemática de reinserción social a partir de la integración de un conjunto de variables asociadas a diversos niveles ecológicos. En función de los resultados obtenidos es posible considerar los siguientes aspectos de mayor relevancia:

(i) Desde el nivel micro, una de las variables que ha demostrado tener un importante papel, refiere al tipo de respuestas de afrontamiento implementadas ante situaciones percibidas como estresantes en el período de transición cárcel-libertad. De acuerdo al análisis de regresión realizado, las estrategias de afrontamiento por aproximación, en particular las basadas en el método conductual, resultaron ser buenos predictores de la reinserción social. En consonancia con otros estudios, puede afirmarse que la implementación de este tipo de estrategias mejora la capacidad de los sujetos para 
enfrentarse con los problemas cotidianos de su vida diaria actual y futura. Asimismo, promueve la adquisición de distintas habilidades para la reconstrucción vital y de los vínculos sociales en el proceso de integración al medio social (Mikulic, 1998; Carr \& Vandiver, 2001; Mohino, Kirchner \& Forns, 2004). Este tipo de estrategias ha sido asociado con el desistimiento delictivo, demostrando ser un factor de protección que discrimina entre quienes vuelven a reincidir luego de una condena y quienes no reinciden (Carr \& Vandiver, 2001; Mikulic \& Crespi, 2007; Blasco Romera, 2012).

(ii) Otra variable de importancia que hemos incluido dentro de este nivel de análisis refiere a la perspectiva temporal futura (PTF). Tal como señalan diversos autores, la dimensión de futuro ocupa un lugar central en la construcción cotidiana de la identidad (Dubar, 2002; Díaz, 2006). La orientación o proyecto de futuro representa una construcción subjetiva que habilita la planificación, exploración y movilización de acciones en torno al logro de metas y objetivos (Nurmi, 1994). Al estudiar la asociación de esta variable con la reinserción social del liberado se ha podido apreciar que, cuanto mayor es la cantidad de metas de futuro que los liberados identifican y fundamentalmente, cuanto mayor es la factibilidad de las mismas, mayores son los indicadores ligados a una adecuada reinserción social. Fundamentalmente, a través del análisis de regresión realizado se ha podido identificar que la actitud temporal, en particular, la actitud positiva hacia el futuro, constituye la dimensión de la PTF que mejor predice las posibilidades de reinserción social.

Asimismo, los resultados permiten concluir que quienes experimentan mayor nivel de satisfacción vital, son quienes presentan mayores indicadores de una reinserción social efectiva y por lo tanto, una mayor integración al medio social tras el egreso carcelario. Esta afirmación se corresponde con los hallazgos de distintas investigaciones que ubican la variable satisfacción vital como un factor personal presente en mayor medida, en quienes no vuelven a cometer delitos luego de una condena (Heisel \& Flett 2004; Kim \& Kim 2008; Martin \& Stermac, 2010; Fulya \& Yasin, 2012; Ward, Rose \& Willis, 2012).

(iii) En consonancia con lo reportado en estudios anteriores, se ha podido advertir que cuanto menor es la edad en la que se comienza a delinquir, menor es la reinserción al 
medio social del sujeto tras la liberación, y por lo tanto, mayores las condiciones de riesgo que pueden conducir a la comisión de nuevos delitos (Vázquez, 2003; Bottoms\& Shapland, 2011; Piquero, Hawkins, Kazemian, Petechuk \& Redondo, 2013).

(iv) Desde el nivel relacional abordado, el apoyo social representa un recurso importante en el marco del proceso de reinserción. De acuerdo al análisis de correlación efectuado, el tamaño de la red se asocia positivamente a la reinserción social. Como señalan diversos autores, se advierte que, cuanto mayor es la cantidad de miembros que conforman la red del sujeto, mayores resultan ser las posibilidades de integración social del mismo (Girard, 2002; Grossetti, 2005). Respecto al nivel funcional, se sostiene la importancia que el vínculo positivo, especialmente con miembros del contexto familiar, tiene en la reinserción social. Ello guarda asidero con lo reportado por diferentes autores que reconocen el apoyo familiar como un factor central en el proceso de reintegración del liberado (Casey \& Jarman 2011; Visher \& Travis, 2011; Cid \& Martí; 2012). Si bien, coincidimos en señalar la relevancia que este constructo tiene en la problemática objeto de estudio, un dato a tener en cuenta es que del conjunto de variables incluidas, el apoyo social ha sido la de menor poder explicativo. Al respecto, podría resultar relevante en futuras investigaciones, analizar el rol que la misma puede tener como variable moderadora en el proceso de reinserción. Como se ha puesto en evidencia en el abordaje de otras problemáticas, es probable que el apoyo social constituya un factor capaz de producir efectos sobre la relación entre dos o más variables. Por ejemplo, en una investigación realizada al estudiar la asociación entre reinserción social y las variables satisfacción vital, apoyo social y afrontamiento, se advirtió que el apoyo social tenía efectos directos positivos sobre la satisfacción vital y el afrontamiento activo e indirectos, a través de esas variables, sobre la reinserción social (Crespi, 2012).

Por otro lado, hay que tener en cuenta que en este estudio se realizó un análisis transversal del apoyo social de los liberados, pretendiendo evaluar su relación con la reinserción. En tal sentido, sería importante poder analizar y comprender la relevancia que la variable apoyo tiene, considerando todo el proceso que representa la transición cárcel-libertad. De aquí se deriva que podemos utilizar dos aproximaciones complementarias para estudiar el apoyo social en el contexto de la reinserción social: por un lado, desde una perspectiva estática evaluando el rol del apoyo con sus 
características actuales; y por el otro, desde un enfoque longitudinal y cualitativo, analizando los cambios que se van produciendo en la estructura y las funciones del apoyo, como resultado de las transiciones ecológicas que representan tanto la privación de libertad como el egreso carcelario.

(v) Por último, al estudiar la percepción de ser objeto de prejuicio, se ha podido advertir la significativa importancia que este factor tiene en la comprensión de la problemática de reinserción social. Considerando la totalidad de variables estudiadas, el análisis de regresión lineal efectuado permitió concluir que la percepción de ser objeto de prejuicio por parte de la sociedad, es el factor que mejor predice la reinserción social de los sujetos en libertad. Diversos estudios apoyan estos resultados al poner en evidencia que la falta de apoyo comunitario y la presencia de actitudes sociales negativas hacia el exrecluso promueven mayor violencia y la percepción de menores oportunidades de inserción (Ross \& Richards, 2003; Comenarez, 2005). En otra investigación, al comparar la percepción de ser objeto de prejuicio entre sujetos que cometieron delitos y sujetos no infractores, se reportaron valores significativamente más altos en los primeros (Valdenegro, 2005). Estas diferencias exponen la relevancia que tiene, tanto para la etiología como para el mantenimiento del fenómeno delictual, el ser percibido y rotulado por los actores sociales como pertenecientes al estereotipo de "delincuentes".

\subsection{Limitaciones}

(i) Contemplando las limitaciones del estudio, se ha intentado a través de este trabajo mostrar algunas de las facetas de un problema mucho más vasto, cuyas manifestaciones no agotan el espectro de factores estudiados. Son apenas indicios de una multiplicidad de variables que exigen nuevas propuestas y desarrollos investigativos. Las cuestiones de género; el abordaje de otros tipos de delitos (no sólo contra la propiedad); la realización de estudios longitudinales que impliquen el seguimiento a largo plazo del liberado; la inclusión de nuevas variables en el análisis del proceso de reinserción; se tornan algunos aspectos que no han podido ser contemplados, alentando la realización de futuras investigaciones. 
(ii) En este estudio no se pretendió abordar en su totalidad las variables asociadas a la reinserción social, ya que por su multiplicidad, esto hubiese dificultado el análisis e interpretación de los datos. En su lugar, se optó por profundizar sobre variables que, desde un enfoque ecológico, ofrecieran información relevante sobre la reinserción. Reconocemos en este punto, la necesidad de incorporar nuevos indicadores de los diversos niveles ecológicos considerados. En especial del nivel macro, contemplando no sólo variables percibidas (como la percepción de prejuicio), sino también objetivas, como las prácticas institucionales, las políticas gubernamentales, la oferta laboral, etc.

(iii) Otra de las limitaciones del estudio efectuado responde al tamaño reducido de la muestra y a su concentración en torno a Unidades del Servicio Penitenciario Federal Argentino. Por tal motivo, los datos obtenidos son válidos dentro del ámbito local, por lo que ofrecen limitaciones a la hora de generalizar los resultados a poblaciones de contextos socioculturales diferentes.

(iv) Por otro lado, en el marco del presente estudio se trabajó con personas que se encontraban en situación de libertad condicional. Podría ser relevante evaluar si la condición jurídica actual del sujeto (libertad condicional, asistida, agotamiento de la pena, entre otras), tiene injerencia en términos del comportamiento del sujeto al obtener su libertad.

(v) Por último y fundamentalmente, tal como se expresó al comienzo de este estudio, la problemática de reinserción social es un fenómeno complejo y multidimensional. Esto hace que no pueda ser estudiada en su totalidad, ni que pueda ser explicada en términos causales, en tanto que, causas y efectos se mezclan a través de un proceso de retroalimentación. Si bien, basándonos en el estado del arte, se ha estudiado la asociación y peso relativo de un conjunto de variables en la reinserción social, ello no significa que existan relaciones lineales o biunívocas entre las mismas. Un ejemplo de ello lo representa la relación que puede establecerse entre satisfacción vital y reinserción social. Estudios previos explican el efecto de la variable satisfacción vital destacando dos aspectos de relevancia: por un lado, el hecho de experimentar mayor satisfacción vital se asocia con la presencia de conductas positivas durante el período de egreso 
carcelario -ligadas a cambios en el estilo de vida y a la búsqueda de inserción en espacios laborales, educativos y/o recreativos-.

Por otro lado, la mayor integración y participación social de los sujetos favorece el sentimiento de autoeficacia y con ello, la satisfacción experimentada con la propia vida (Damon, 2004; Catalano, Berglund, Ryan, Lonczak \& Hawkins, 2004). La misma relación bidireccional puede plantearse en el caso del tamaño de la red o del apoyo social y la reinserción. En la medida en que el sujeto se va insertando en nuevos espacios sociales y laborales, se va incrementando el tamaño de su red y sus recursos de apoyo. Por tal motivo, resulta necesario en futuras investigaciones, la inclusión de nuevos diseños metodológicos que permitan captar las relaciones recíprocas existentes entre múltiples variables. La metodología de ecuaciones estructurales (SEM) puede ser particularmente útil en este sentido. En efecto, una de las diferencias con respecto a los modelos de regresión múltiple, es que permite captar el interjuego (feedback) entre las variables objeto de estudio, además de poner a prueba modelos teóricos integrales (Batista \& Coenders 2000).

\subsection{Implicaciones}

Para finalizar, considerando los aportes efectuados, si bien se requieren nuevos estudios que arrojen mayor visibilidad a la problemática abordada, los resultados obtenidos tienen importantes implicaciones prácticas para la intervención psicosocial ligada a la problemática de reinserción. Ello, en la medida en que se han podido identificar diversas variables que están directamente implicadas, positiva o negativamente, con la misma. En esta línea, el enfoque ecológico proporciona una guía para la propuesta de sistemas de intervención. De acuerdo a ello, se requieren programas de intervención y políticas integrales, capaces de dar respuestas en los distintos niveles en los que se expresa el proceso de reinserción social. Intervenir es potenciar, y para potenciar es preciso fomentar la utilización de los recursos disponibles o desarrollar prácticas que permitan el acceso a los mismos (Zimmerman, 2000). Los mecanismos de fortalecimiento deben extender el campo de las acciones posibles, tanto desde el punto de vista de los recursos personales como los del entorno, favoreciendo dinámicas de participación e inclusión comunitaria. 


\section{Agradecimientos}

Un agradecimiento muy especial al Consejo Nacional de Investigaciones Científicas y Técnicas-CONICET y al Centro Interdisciplinario de Investigaciones en Psicología Matemática y Experimental-CIIPME, por el apoyo recibido y por posibilitar el desarrollo de este estudio. Asimismo, agradecemos a los evaluadores de la REIC por sus valiosas observaciones y comentarios al presente manuscrito.

\section{Financiación}

El estudio que se presenta en este artículo ha sido realizado en el marco de una beca de investigación otorgada por el Consejo Nacional de Investigaciones Científicas y Técnicas-CONICET.

\section{Referencias}

Angenent, H. \& Man, A. (1996). Background factors of juvenile delinquency. New York: Peter Lang Publishing.

Bain, A. \& Parkinson, G. (2010). Resettlement and social rehabilitation: Are we supporting success? Probation Journal, 57(1), 63-74.

Batista, J. \& Coenders, G. (2000). Modelos de Ecuaciones Estructurales. Madrid: La Muralla.

Belsky, J. (1993). Etiology of child maltreatment: a developmental-ecological analysis. Psychological Bulletin, 114(3), 413-434.

Bisquerra, R. (1989). Métodos de investigación educativa. Barcelona: CEAC.

Black, W. \& Grecson, R. (2011). Time perspective, purpose in life, extraversion and neuroticism in New Zealand prisoners. British Journal of Social and Clinical Psychology, 12(1), 50-60.

Blasco, C. (2012). Descripción y análisis de los factores protectores de adolescentes en la prevención del delito. Barcelona: Centro de Estudios Jurídicos y Formación Especializada.

Bottoms, A. \& Shapland, J. (2011). Steps towards desistance among male young adult recidivists. En S. Farrall, M. Hough, S. Maruna \& R. Sparks (Eds), Escape routes: contemporary perspectives on life after punishment. London: Routledge.

Bringas, C., Rodríguez, F., Moral, M., Pérez, S. \& Ovejero, A. (2012). Comportamiento delictivo reincidente. Análisis diferencial de la variable edad. Revista Interamericana de Psicología, 46(3), 365-374. 
Bronfenbrenner, U. (1987). Ecología del desarrollo humano. Barcelona: Paidós.

Camarillo, J. M. \& García, P. (2003). Los métodos de regresión múltiple. Disponible en http://www.Tesisenxarxa.net.

Carr, M. \& Vandiver, T. (2001). Risk and protective factors among offenders. Adolescence, 36(43), 409-426.

Casey, J. \& Jarman, B. (2011). The social reintegration of ex-prisoners in council of Europe member states. Brussels: Quaker Council of European Affairs.

Castellá, J. (2008). El paradigma ecológico en la psicología comunitaria: del contexto a la complejidad. En E. Saforcada \& J. Castellá (Comps.), Enfoques conceptuales y técnicos en Psicología Comunitaria. Buenos Aires: Paidós.

Catalano, R., Berglund, M., Ryan, J., Lonczak, H., \& Hawkins, J. (2004). Positive youth development in the United States: research findings on evaluations of positive youth development programs. The Annals of American Academy of Political \& Social Science, 591(1), 98-124.

Céspedes, G. (2005). La nueva cultura de la discapacidad y los modelos de rehabilitación. Aquichán, 5(1), 108-113.

Cid, J. \& Martí, J. (2011). El proceso de desistimiento de las personas encarceladas. Obstáculos y Apoyos [Informe de investigación]. Barcelona, Centre d'Estudis Jurídics i Formació Especialitzada. Disponible en http://www20.gencat.cat/docs/Justicia/Documents/ARXIUS/SC_3_175_11_cast. pdf

Cid, J. \& Martí, J. (2012). Turning points and returning points: Understanding the role o family ties in the process of desistance. European Journal of Criminology, 9(6), 603-620.

Clinkinbeard, S. (2013). What Lies Ahead: An Exploration of Future Orientation, SelfControl, and Delinquency. Criminal Justice Review, 1, 1-18.

Colmenarez, J. (2005). Actitud familiar ante la llegada del liberado al hogar. Capítulo Criminológico, 33(1), 79-113.

Crespi, M. (2012). Transición cárcel-libertad: estudio del proceso de reinserción social en liberados condicionales desde el enfoque ecológico-social. Tesis doctoral inédita. Facultad de Psicología, Universidad de Buenos Aires, Argentina.

Crespi, M. \& Mikulic, I. (2004). Calidad de vida percibida: un estudio de los factores de riesgo y protección en sujetos privados de libertad. Memorias de las XI Jornadas de Investigación, 2, 350-353. Universidad de Buenos Aires. 
Damon, W. (2004). What is positive youth development? ANNALS, American Academy of Political and Social Science, 591(1), 13-24.

Díaz, J. (2006). Perspectiva temporal futura y ciclo vital. Anales de Psicología, 22(1), 52-59.

Dodge, M. \& Pogrebin, M. (2001). Collateral costs of imprisonment for women: complications of reintegration. The Prison Journal, 18(1), 42-54.

Dubar, C. (2002). La crisis de las identidades. La interpretación de una mutación. Barcelona: Ed. Bellaterra.

Frías, A., López, E., \& Díaz, S. (2003). Predictores de la conducta antisocial juvenil: un modelo ecológico. Estudos de Psicologia, 8(1), 15-24.

Fulya, O. \& Yasin, Y. (2012). The role of life satisfaction and parenting styles in predicting delinquent behaviors among high school students. Educational Sciences: Theory \& Practice, 12(3), 17-44.

García, C. (1991). ¿A dónde va la prisión? Doctrina y Acción Postpenitenciaria, 5(7), 15-56.

Girard, F. (2002). Políticas públicas y tecnología: el Comité Nacional para la Sociedad de la Información. Un proyecto en red. Colección de Documentos. Instituto Internacional de Gobernabilidad. Disponible en http://www.iigov.org/documentos/?p=6_0086

Green, S. (1991). How many subjects does it take to do a regression analysis? Multivariate Behavioral Research, 26(2), 499-510.

Grossetti, M. (2005). Where do social relations come from? A study of personal networks in the Toulouse area of France. Social Networks, 27(4), 289-300.

Heisel, M. \& Flett, G. (2004). Purpose in life, satisfaction with life, and suicide ideation in a clinical sample. Journal of Psychopathology and Behavioral Assessment, 26(2), 127-135.

Ibañez, A. \& Cid, J. (2013). El rol de la comunidad en la reinserción. Ponencia presentada en el XI Congreso Español de Sociología, Universidad Complutense de Madrid.

Kelly, J. (1992). Contextos cambiantes y el campo de la Psicología Comunitaria. En E. Saforcada (Ed.), Psicología Comunitaria: el enfoque ecológico contextualista de James Kelly. Buenos Aires: Proa XXI.

Kim, H. \& Kim, H. (2008). Risks factors for suicide attempts among adolescents. Child Psychiatry and Human Development, 39(3), 221-235. 
Laparra, M. (1999). El espacio social de la exclusión: el caso de Navarra. Madrid: UNDED Ediciones.

Laub, J. \& Sampson, R. (2003). Shared beginnings, divergent lives. Cambridge, MA: Harvard University Press.

Laws, D. \& Ward, T. (2011). Desistance from sexual offending: Alternatives to throwing away the keys. New York: Guilford Press.

Lösel, F. (2010). What works in offender rehabilitation: A global perspective. Trabajo presentado en 12th Annual Conference of the International Corrections and Prisons Association, Ghent, Belgium.

Martin, K. \& Stermac, L. (2010). Measuring hope: Is hope related to criminal behaviour in offenders? International Journal of Offender Therapy and Comparative Criminology, 54(5), 693-705.

Martínez, P. (2004). Perspectiva temporal futura y satisfacción con la vida a lo largo del ciclo vital. Revista de Psicología de la Pontificia Universidad Católica del Perú, 22(2), 215-252.

Mikulic, I. \& Crespi, M. (2007). Aportes de la Entrevista ERA a la evaluación psicológica de factores de riesgo y protección y potencial resiliente. Buenos Aires: Departamento de Publicaciones de la Facultad de Psicología, UBA.

Mikulic, I.M. (1998). Ecoevaluación Psicológica del ex delincuente en su ambiente natural. Tesis doctoral inédita. Universidad de Belgrano, Argentina.

Mohino, S., Kirchner, T., \& Forns, M. (2004). Coping strategies in young male prisoner. Journal of Youth and Adolescent, 33(1), 41-50.

Moos, R (1993). Coping Responses Inventory Psychological Assessment Resources. Odessa: Psychological Assessment Resources, Inc.

Nunnally, J. (1991). Teoría psicométrica. México: Trillas.

Nurmi, J. (1994). The development of future orientation in life-span context. En Z. Zaleski (Ed.), Psychology of future orientation. Lublin: Towarzystwo Naukowe.

Pavot, W. \& Diener, E. (1993). Review of the Satisfaction with Life Scale. Psychological Assessment, 5(2), 164-172.

Piquero, A., Hawkins, D., Kazemian, L., Petechuk, D. \& Redondo, S. (2013). Transición desde la delincuencia juvenil a la delincuencia adulta. Revista Española de Investigación Criminológica, 1, 11. Disponible en http://www.criminologia.net

Prilleltensky, I. (2000). Community Psychology. Nueva Zelanda: McMillan Press. 
Rodgers, B. (2009). An ecological approach to understanding the stigma associated with receiving mental health services: the role of social proximity. Tesis doctoral, Faculty of Virginia Polytechnic Institute and State University.

Ross, J. \& Richards, S. (2003). Convict Criminology. Belmont, CA: Wadsworth.

Sobsey, D. (1994). Violence and abuse in the lives of people with disabilities: The end of silent acceptance? Baltimore: Brookes.

Tabachnick, B. \& Fidell, L. (2001). Using multivariate statistics. New York: Harper \& Row.

Torrico, E., Santín C., Monserrat, A., Menéndez, S. \& López, M. (2002). El modelo ecológico de Bronfenbrenner como marco teórico de la psicooncología. Análisis de la Psicología, 18(1), 45-59.

Trommsdorff, G. \& Lamm, H. (2006). Future orientation of institutionalized and noninstitutionalized delinquents and nondelinquents. European Journal of Social Psychology, 10(3), 247-278.

Trommsdorff, G. (1994). Future time perspective and control orientation: Social conditions and consequences. Lublin, Poland: Towarzystwo Naukowe KUL.

Uggen, C., Manza, J., \& Behrens, A. (2004). Less than the average citizen: Stigma, role transition and the civic reintegration of convicted felons. En S. Maruna \& R. Immarigeon (Eds.), After crime and punishment: Pathways to offender reintegration. London: Willan.

Valdenegro, B. (2005). Factores psicosociales asociados a la delincuencia juvenil. Psykhe, 14(2), 33-42.

Vázquez, C. (2003). Teorías criminológicas sobre delincuencia juvenil. En Colex (Ed.), Delincuencia juvenil. Consideraciones penales y criminologicas. Madrid, España.

Villagra, C. (2008). Hacia una política pospenitenciaria en Chile. Desafíos para la reintegración de quienes salen de la cárcel. Chile: Ril.

Visher, C., \& Travis, J. (2011). Life on the outside: Returning home after incarceration. The Prison Journal, 91(3), 102-119.

Ward, T., Rose. C. \& Willis, G. (2012). The rehabilitation of offenders: Good lives, desistance, and risk reduction. En G. Davis \& A. Beech (Eds.), Forensic Psychology: Crime, Justice, Law, Interventions, Oxford, UK: Wiley Blackwell.

Zimmerman, M. (2000). Empowerment theory. En J. Rappaport \& E. Seidman (Eds), Handbook of community psychology. New York: Kluwer. 
Melina Crespi ha recibido los títulos de: Doctora en Psicología, Magíster en Psicología Social Comunitaria y Licenciada en Psicología de la Universidad de Buenos Aires. Es becaria de investigación posdoctoral del Consejo Nacional de Investigaciones Científicas y Técnicas-CONICET e investigadora formada UBACyT. Se desempeña como coordinadora docente de la Maestría en Psicodiagnóstico y Evaluación Psicológica y como docente adjunta de la Facultad de Psicología de la Universidad de Buenos Aires en las asignaturas 'Teoría y Técnica de Exploración y Diagnóstico Psicológico“ y “Práctica de Investigación en Evaluación Psicológica en Contexto“.

Isabel M. Mikulic es Doctora en Psicología, Licenciada en Psicología y Asistente Social. Trabaja como Secretaria de Posgrado y Miembro Titular de la Comisión de Doctorado de la Facultad de Psicología de la Universidad de Buenos Aires. Es directora de la Maestría en Psicodiagnóstico y Evaluación Psicológica y Profesora Titular de la Facultad de Psicología de la Universidad de Buenos Aires en las asignaturas 'Teoría y Técnica de Exploración y Diagnóstico Psicológico" y "Práctica de Investigación en Evaluación Psicológica en Contexto“. Es Directora de Proyectos de Investigación UBACyT e investigadora Categoría I, Ministerio de Educación de la Nación. Es cofundadora y tesorera de la International Society for Emotional Intelligence. 